\title{
Competencia digital en la auditoría. Soporte o carga en el ejercicio profesional de los auditores*
}

doi:10.11144/Javeriana.cc15-37.cdas

\section{Fabio Enrique Gómez-Meneses}

Contador público y especialista en revisoría fiscal y auditoría externa, Universidad Autónoma de Bucaramanga, UNAB. Master en administración electrónica de empresas y master en contabilidad y finanzas, Universidad de Zaragoza, España. Doctor en contabilidad y finanzas, Universidad de Zaragoza, España. Profesor investigador de tiempo completo de la Facultad de Ciencias Económicas y Contables de la Universidad Autónoma de Bucaramanga, UNAB. Director del grupo Investigación en Contabilidad y Finanzas, INVESCONFIN.

Correo electrónico: fgomezm@unab.edu.co

\footnotetext{
* Artículo derivado del proyecto de investigación Competencia digital en la auditoría. Soporte o carga en el ejercicio profesional de los auditores, financiado por la Universidad Autónoma de Bucaramanga, UNAB, y ejecutado durante 2013. Código del Proyecto: I-12093.
} 
Resumen Las tecnologías de la información y la comunicación (TIC) son, en la actualidad, una invaluable herramienta en el desarrollo de todas y cada una de las actividades que ejecutan las personas y las empresas. La contaduría pública como profesión no ha sido ajena a esta influencia, desde el uso de simples aplicaciones contables y de auditoría en pequeñas empresas, hasta los más complejos sistemas ERP (Enterprise Resource Planning) para el manejo y revisión de información contable y financiera de grandes compañías. Teniendo en cuenta este contexto, en el presente trabajo se caracteriza el nivel de competencia tecnológica de una muestra de auditores de la ciudad de Bucaramanga en el ejercicio de sus funciones, para analizar si las TIC son un soporte o carga en su devenir profesional. Infortunadamente, los resultados encontrados en la investigación, respecto al nivel tecnológico de los auditores, son negativos, en contraste con el horizonte tecnológico evidenciado en las empresas donde estos profesionales se desempeñan.

Palabras clave autor: auditoría; tecnologías de la información y la comunicación; competencia digital; profesión contable

Palabras claves descriptor: auditoría; tecnologías de la información y la comunicación; contaduría como profesión

\section{Códigos JEL: M42}

\section{Digital Competence in Auditing. Support or Burden on the Professional Practice of}

\section{Auditors}

\footnotetext{
Abstract Information and communication technologies (ICT) are currently a priceless tool in the development of each and every single activity performed by people and companies. Public accounting, as a profession, has not been a stranger to this influence: from the use of the simplest accounting and auditing applications in small companies, to
}

the most complex Enterprise Resource Planning (ERP) systems for management and reviewing of accounting and financial information of large companies. Taking this context into account, in this work the technological competence level of a sample of auditors, in the course of their duties, in the city of Bucaramanga, is characterized, to analyze whether ICT's are a support or a burden in their professional development. Unfortunately, the results found in the course of the research are negative, regarding the technological level of the auditors, contrasting the technological horizon of the companies where these professionals work.

Key words author: auditing; information and communication technologies; digital competence; accounting profession

Key words plus: auditing; information and communications technologies; accounting; accounting as profession

\section{Concorrência digital na auditoria. Suporte ou carga no exercício profissional de auditores}

Resumo As tecnologias da informação e comunicação (TIC) são, na atualidade, valiosa ferramenta no desenvolvimento de todas e cada uma das atividades executadas pelas pessoas e empresas. A Contabilidade Pública como profissão não ficou imune dessa influência, desde o uso de simples aplicações contábeis e de auditoria em pequenos negócios até os mais complexos sistemas ERP (Enterprise Resource Planning) para o maneio e revisão de informação contábil e financeira de grandes companhias. Levando em conta tal contexto, no presente trabalho é caraterizado o nível de concorrência tecnológica de uma amostra de auditores da cidade de Bucaramanga no exercício das suas funções, para analisar se as TIC são suporte ou carga no seu devir profissional. Infelizmente, os resultados encontrados na pesquisa no que diz respeito ao nível tecnológico dos auditores são negativos, em contraste com o horizonte 
tecnológico evidenciado nas empresas em que eles se desempenham.

Palavras-chave autor: auditoria, tecnologias da informação e comunicação; concorrência digital; profissão contábil

Palavras-chave descritor: auditoria; tecnología da informação e comunicação; contadores

\section{Introducción}

La gran mayoría de empresas, independientemente de su tamaño, lleva a cabo sus actividades financieras y contables mediante programas y aplicaciones informáticas; lógicamente, todo este trabajo debe ser revisado y evaluado, en otras palabras, debe estar sujeto a un proceso de auditoría, para garantizar que la información emitida por una organización corresponda fielmente a su realidad económica. En este contexto, la auditoría cobra un papel protagónico, en particular, la auditoría financiera, pues garantiza tres aspectos fundamentales: la razonabilidad de los estados financieros, el cumplimiento de las normas aplicables y la uniformidad en la aplicación de tales normas.

Resulta lógico que si las compañías utilizan masivamente las TIC en sus actividades, la función de auditoría debería hacer lo propio en el desarrollo de su trabajo; si esto es así, el auditor deberá tener un amplio conocimiento sobre una gran variedad de técnicas automatizadas, programas y procedimientos de auditoría, que garanticen que la labor realizada tenga la mayor calidad posible, de acuerdo con lo establecido por los más importantes estándares internacionales vigentes, como las Nor- mas Internacionales de Auditoría, NIA, y las Normas Internacionales de Aseguramiento de la Información. Infortunadamente, en algunas ocasiones, no siempre los auditores tienen este conocimiento, situación que puede generar una serie de efectos negativos en la calidad del trabajo desarrollado por el auditor y, por añadidura, en el buen desempeño de la empresa evaluada.

A esta falta de preparación de algunos auditores, hay que sumarle la situación actual de esta importante función de la profesión contable. Es evidente que el ejercicio profesional de la auditoría ha estado en entredicho en los últimos años; según María Antonia GarcíaBenau y Antonio Vico-Martínez (2003), la auditoría desde hace ya varios años atraviesa una profunda crisis de confianza, crisis que se ha generado por los múltiples y sonados casos de fraude empresarial, tanto nacionales como internacionales y, más recientemente, por la actual crisis económica de las principales economías del planeta. Estas situaciones de fraude y crisis, en ocasiones, son pasadas por alto por los auditores que, aunque no forman parte de su responsabilidad principal en la ejecución de un trabajo de auditoría, según lo establecido en las NIA, no pueden dejarlas a un lado, ya que afectan la calidad de la información de las organizaciones. Algunas de estas inconsistencias no son descubiertas por los auditores, tal vez por su precaria formación tecnológica, lo cual estimula la comisión de fraudes y de situaciones indeseables para las organizaciones con el uso de tecnologías informáticas.

De lo expuesto anteriormente pueden surgir múltiples interrogantes. Por ejemplo, ¿los 
procesos de formación en la academia están siendo lo suficientemente rigurosos?, ¿las normas y leyes aplicables en el entorno profesional y empresarial son las que se requieren?, ¿se están llevando a cabo adecuados procesos de normalización internacional en el ámbito de la auditoría? Todas estas cuestiones habrá que analizarlas en detalle, pero en el contexto de lo que supone la ejecución de este trabajo, se desarrollarán otras dos en particular: ¿cuál es el nivel de preparación tecnológica que tienen los auditores de la ciudad de Bucaramanga en su ejercicio profesional? y ¿este nivel resulta pertinente en el contexto tecnológico en el cual funcionan las empresas sujetas a los procesos de auditoría?

Específicamente, y de acuerdo con lo establecido en los párrafos precedentes, en el presente documento se caracteriza el nivel tecnológico de una muestra de auditores de la ciudad de Bucaramanga en su ejercicio profesional, de tal forma que se pueda establecer si el grado de preparación tecnológica de la población de auditores evaluada, responde a las necesidades que plantea el actual entorno tecnológico, en el cual se desempeñan muchas de las empresas sujetas a procesos de auditoría; todo ello en el contexto mundial de crisis económica y fraudes corporativos que en muchas ocasiones se ve impulsado o facilitado por el uso de herramientas informáticas.

Los resultados obtenidos servirán de insumo para el desarrollo de la profesión contable, en particular, en el ejercicio de la auditoría, ya que se podrán identificar las posibles debilidades tecnológicas de los auditores en algunos aspectos fundamentales, como la capacidad de reconocimiento y tratamiento de la evidencia digital, situación que limita la respuesta del profesional para mitigar situaciones de fraude o crisis económica y, que por su relevancia, ya ha sido estudiada en trabajos previos (Gubba, Gutfraind, Rodríguez \& Villamarzo, 2001).

Con el resultado del trabajo efectuado, se podrán diseñar, construir e implementar, desde la academia y con la participación de organismos de la profesión, como el Consejo Técnico de la Contaduría Pública, CTCP, iniciativas como cursos, programas de actualización y diplomados en TIC aplicados al ámbito de la auditoría, de manera que el uso de las tecnologías de la información y la comunicación por parte de los auditores, no se convierta en una carga, sino en un pilar que fortalezca el ejercicio profesional.

\section{Marco teórico}

Desde hace varios siglos, la auditoría es una actividad que se ha venido desarrollando en las organizaciones y sus objetivos han venido evolucionando con el paso de los años. Desde el siglo XV y hasta el siglo XIX, la auditoría estuvo enfocada principalmente a la búsqueda y detección de fraudes, pero a partir de la primera mitad del siglo XX, los objetivos de la auditoría cambiaron y esta función de la profesión contable se centró en determinar la veracidad de la situación financiera y de los resultados de las operaciones, y dejó en un segundo plano la búsqueda y detección de fraudes.

Durante las décadas de 1940 y 1950, según lo planteado por W. Thomas Porter y John Burton (1980), la auditoría se enfocó en la re- 
visión de los sistemas de control interno en las empresas. A medida que las organizaciones iban creciendo, también aumentaba la dificultad de realizar trabajos de auditoría, debido a la cantidad y la complejidad de las operaciones; por tanto, según lo expuesto en el trabajo de María del Carmen Martínez e Ileana Armenteros (2006), las compañías comenzaron a tener dependencias o divisiones en diversos lugares y se creó un organismo en la propia empresa que se encargaba de verificar la protección de los activos, la fiabilidad de la información y el cumplimiento de las políticas y procedimientos establecidos para la detección y prevención de fraudes.

En la segunda mitad del siglo XX, la función de la auditoría se ha dirigido principalmente a apoyar a las compañías en la solución de problemas relacionados con la dirección y gestión empresarial, en busca de la eficiencia y la eficacia en las operaciones que realizan las organizaciones.

Finalmente, en las últimas dos décadas, y gracias a la evolución económica, política y social de los países, se ha generado una serie de nuevos retos y condiciones comerciales para el desarrollo de los objetos sociales de las empresas, retos que exigen el uso de las tecnologías de la información y la comunicación, como el uso de internet para la revelación y divulgación de la información empresarial o la utilización de sistemas de facturación electrónica.

Estas y otras tecnologías de la información y la comunicación han modificado sustancialmente la forma como las empresas llevan a cabo sus actividades. Los computadores, las ba- ses de datos, los programas informáticos, internet y el comercio electrónico son algunas de las muchas TIC que las compañías utilizan en la actualidad.

Con todas ellas, las empresas esperan lograr beneficios, como la reducción de costos y tiempos en los procesos, la obtención de ventajas competitivas y la mejora en el manejo y administración de la información de las organizaciones, como bien indican Wenhong Luo, David Cook, Jimmie Joseph y Bopana Ganapathy (2000), Anitesh Barua, Charles Kriebel y Tridas Mukhopadhyay (1995) y Marcos Valderrama (2002), respectivamente.

Partiendo del concepto de TIC que, según Fabio Gómez (2010), se definen como “todas aquellas herramientas de carácter computacional e informático que sustentan y automatizan los procesos de entrada, almacenamiento, uso y presentación de la información mediante nuevos medios que faciliten su comunicación a los diferentes interesados en ella", desde una óptica empresarial, la función esencial de las tecnologías de la información y la comunicación es la de incrementar la capacidad de los trabajadores de creación, distribución y procesamiento de la información, para aumentar el crecimiento económico de las organizaciones.

Específicamente, en el ámbito de lo contable y financiero, también podemos reseñar tecnologías que afectan trascendentalmente las operaciones empresariales, los ERP (Enterprise Resource Planning), el EDI (Electronic Data Interchange), la facturación electrónica y el reporte financiero por internet, todas ellas ampliamente estudiadas y analizadas en la literatura científica (Gómez, 2010; Lorca \& De Andrés, 
2007; Gómez \& Católico, 2009; Católico \& Gómez, 2013).

Infortunadamente, el uso de la tecnología no solo significa beneficios y ventajas para las empresas, sino que también en ocasiones, es foco de problemas para las compañías. Stephen S. Roach (1991), Alain Pinsonneault y Suzanne Rivard (1998) y Erik Brynjolfsson (1993) evidenciaron descensos en la productividad de los trabajadores en empresas que aplicaban TIC en sus operaciones; en su trabajo Pedro LorcaFernández y Juan de Andrés-Suárez (2007) comprobaron deterioros significativos en la rentabilidad financiera y económica de las empresas al implementar sistemas ERP.

En este mismo sentido, es importante destacar que el uso de TIC en las organizaciones, en ocasiones, facilita la comisión de actos fraudulentos e ilícitos que pueden afectar la estabilidad financiera y contable de una compañía, aunque también es cierto que se multiplican las evidencias digitales de tales actuaciones.

En este momento, aparece el auditor como garante de las operaciones que se lleven a cabo en las empresas, independientemente de que estas se ejecuten o no totalmente soportadas en tecnologías de la información y la comunicación. La realidad indica que en muchas compañías, incluso en algunas pequeñas, las operaciones se realizan de forma electrónica, al igual que los fraudes e ilícitos que se ejecutan; por tanto, el auditor debe estar en total capacidad de detectar las "pruebas digitales" que evidencian los fraudes cometidos.

Algunos de estos ilícitos son denominados delitos informáticos, que el Código Penal Colombiano define como "aquellas conductas que recaen sobre las herramientas informáticas propiamente dichas, bien sean programas, computadoras, etc., y aquellas conductas que valiéndose de los medios informáticos lesionan o perjudican los intereses de otras personas o empresas". Parece que en algunos casos los contadores públicos no están preparados para detectar este tipo de situaciones en las organizaciones.

Ahora bien, podría derivarse como conclusión (tal vez, equivocada) ante la gran cantidad de actos fraudulentos e ilícitos que se están presentando en las empresas en los últimos años, que las competencias tecnológicas, las técnicas, las herramientas y los mecanismos de control utilizados por los auditores no tienen el mismo desarrollo tecnológico que las actividades y procesos que son objeto de evaluación en las compañías (Benvenuto, 2004).

La crisis de confianza que está sufriendo la auditoría es inocultable, no solo en nuestro país, sino en el mercado mundial. Esta crisis tiene sus orígenes en los sonados casos de Enron, WorldCom y Parmalat, pero se ha extendido a diversas organizaciones mundiales, lo que ha llevado a la auditoría a experimentar serios problemas, entre los cuales podemos mencionar principalmente tres, según lo expuesto por García y Vico (2003):

- La falta de capacidad en la detección de los fraudes.

- La falta de independencia.

- El no uso de las TIC en el desarrollo de los trabajos de auditoría.

Esta situación plantea un nuevo desafío para el auditor, como bien es descrito en el tra- 
bajo de Enrique Bonsón-Aponte (2000); este desafío exige una adecuada preparación y conocimiento en cuanto a los nuevos procedimientos, controles y riesgos involucrados en empresas que funcionan soportadas en las TIC (Gubba, Gutfraind, Rodríguez \& Villamarzo, 2001); en otras palabras, la auditoría en la economía digital exige un alto grado de formación tecnológica por parte de los auditores.

Igualmente, se requieren procesos de normalización internacional, leyes que sean más rigurosas y estrictas y, en la medida de lo posible, la extensión en la responsabilidad que tiene el auditor, es decir, que en el desarrollo de la función de auditoría, el contador público responda más allá de su informe y sus recomendaciones y entre a verificar la ejecución de todas las sugerencias y observaciones indicadas en el informe de auditoría.

Algunas de estas exigencias han sido consideradas, desde hace ya varios años, por diferentes organismos mundiales, entre los cuales se destaca la IFAC (International Federation of Accountants), con la emisión de sus guías de educación, en las cuales, por ejemplo, reconoce la importancia de las TIC en el trabajo del contador público y plantea una serie de lineamientos como la inclusión de temáticas relacionadas con tecnologías de la información en los planes de estudios para contadores.

$\mathrm{Al}$ respecto, la IFAC - específicamente en sus estándares internacionales de educación para profesionales contables (IFAC, 2009a), en el número 2 (International Education Standards - IES 2) y en el IEPS 2.1 (International Education Practice Statement - Information Technology for Professional Accountants) que es una orientación sobre cómo aplicar lo descrito en el IES 2- plantea que el profesional contable debe tener amplios y suficientes conocimientos generales en tecnologías de la información, pero adicional a ello, debe tener competencias como usuario y controlador de TIC en las organizaciones, y también competencias en la evaluación y diseño de sistemas de información.

En este mismo sentido, es posible mencionar lo expuesto en las Normas Internacionales de Auditoría de la IFAC, más específicamente en la NIA 315 (IFAC, 2009b), que expone la importancia del conocimiento y el buen desempeño que debe tener el auditor con las TIC. La norma establece que el auditor debe obtener un entendimiento de cómo la entidad ha respondido a los riesgos que surgen de la aplicación de TIC, riesgos que, si no se mitigan, podrían resultar en situaciones que comprometerían la estabilidad de una organización.

Un último ejemplo evidencia el interés mundial sobre este tema; es el trabajo realizado por la Information Systems Audit and Control Association (ISACA), organización que ha emitido una serie de estudios, entre los cuales se destaca el informe COBIT, documento en el cual se plantea una estructura de conocimientos y habilidades relacionadas con Sistemas de Información y TIC, en todos los niveles: diseño, construcción, mantenimiento, control y evaluación.

Según lo planteado por estas importantes organizaciones (IFAC e ISACA) en sus diferentes normas y documentos, ya mencionados en los párrafos anteriores, el papel del contador público auditor no se circunscribe específicamente 
a ser un simple usuario de las tecnologías de la información y la comunicación, sino que, por el contrario, se le exige un conocimiento en el rango de diseñador, administrador y evaluador de TIC. En esta exigencia radica el principal reto que los auditores deben enfrentar.

La pregunta lógica que el gremio profesional de la contaduría pública en el país debe hacerse, es si los contadores públicos que se desempeñan como auditores o revisores fiscales tienen el suficiente grado de formación y preparación tecnológica para enfrentar este nuevo paradigma. Por ejemplo, si los profesionales contables disponen de los conocimientos necesarios en técnicas automatizadas de auditoría (datos de prueba, caso básico, etc.), en técnicas para verificar transacciones (paquetes y rutinas de auditoría), en técnicas para analizar programas (traceo, mapeo, comparación de código, etc.), en el uso de estrategias de seguridad, como las firmas digitales, los certificados digitales y la criptografía, por nombrar algunas de ellas. En últimas, se trata de establecer si la incorporación de TIC en los procesos de control y auditoría por parte de los auditores, ha mostrado el mismo nivel de desarrollo frente a la rapidez con la cual se implementan las innovaciones tecnológicas en las empresas.

Todo lo anterior, en el contexto de las exigencias de una sociedad de la información, como la sociedad en la cual estamos insertos, entendiendo el concepto, en palabras de Gómez (2010), como "aquella comunidad en la cual todos los individuos que la conforman tengan fácil acceso a la información y al conocimiento existentes, de tal forma que cada persona se desarrolle plenamente y mejore su calidad de vida, individualmente, pero también de forma colectiva".

Precisamente, este trabajo tratará de solucionar esta y otras cuestiones, mediante un análisis del nivel tecnológico de una muestra de auditores de la ciudad de Bucaramanga, en contraste con el nivel tecnológico de las empresas en las cuales se desempeñan, para establecer si los profesionales contables que laboran en esta importante área de desempeño profesional, lo están haciendo acorde a las mejores prácticas establecidas y en beneficio de las entidades para las cuales trabajan.

Los resultados obtenidos y presentados en este diagnóstico tecnológico permitirán diseñar y proponer iniciativas de formación dirigidas a los contadores/auditores, acciones que podrán ser aplicadas con el concurso y respaldo de organismos contables nacionales, como el Consejo Técnico de la Contaduría Pública, CTCP, para ser coherentes con algunas de las cuestiones tratadas en la Ley 1314 de 2009 y contribuir a mejorar el nivel de preparación tecnológica de los auditores, progreso que también brindará beneficios a las organizaciones empresariales.

\section{Investigaciones previas}

Las investigaciones existentes respecto a la competencia tecnológica de los auditores o trabajos que hayan hecho análisis o mediciones similares son escasas. A pesar de esta insuficiencia de material bibliográfico, es posible reseñar algunos estudios que han analizado esta problemática y que pueden ser de ayuda por si el lector desea profundizar sobre la temática en cuestión. Los trabajos son los siguientes: 


\begin{tabular}{llc}
\hline \multicolumn{1}{c}{ Autores } & \multicolumn{1}{c}{ Título del documento } & Año \\
\hline José Nicolás Gómez & $\begin{array}{l}\text { El fraude y otras amenazas al sistema financiero } \\
\text { latinoamericano. }\end{array}$ & 2010 \\
Jorge Eliécer Ojeda-Pérez, Fernando Rincón- & $\begin{array}{l}\text { Delitos informáticos y entorno jurídico vigente en } \\
\text { Colombia. }\end{array}$ & 2010 \\
$\begin{array}{l}\text { Rodríguez, Miguel Eugenio Arias-Flórez y } \\
\text { Libardo Alberto Daza-Martínez. }\end{array}$ & & 2007 \\
$\begin{array}{l}\text { Daniella Caldana, Ricardo Correa y Héctor } \\
\text { Ponce }\end{array}$ & $\begin{array}{l}\text { Competencias de los auditores gubernamentales chilenos } \\
\text { para la obtención de evidencia electrónica de auditoría. }\end{array}$ & 2004 \\
Ángelo Benvenuto-Vera & $\begin{array}{l}\text { Los delitos informáticos y la función de auditoría } \\
\text { informática. }\end{array}$ & 2003 \\
$\begin{array}{l}\text { María Antonia García-Benau y Antonio Vico- } \\
\text { Martínez }\end{array}$ & $\begin{array}{l}\text { Los escándalos financieros y la auditoría: pérdida y } \\
\text { recuperación de la confianza en una profesión en crisis. } \\
\text { - CICA }\end{array}$ & Electronic Audit Evidence, Research Report. \\
Xavier Llopart-Pérez & Gestión de los recursos humanos en base a competencias. \\
& Análisis de competencias en empresas de auditoría. \\
\hline
\end{tabular}

Cuadro 1. Investigaciones previas sobre competencias tecnológicas de los auditores Fuente: elaboración propia

De estos siete trabajos reseñados, solo uno de ellos está directamente relacionado con los intereses de investigación que se presentan en este documento. El trabajo en cuestión es el Daniella Caldana, Ricardo Correa y Héctor Ponce (2007), estudio en el cual se determinaron las competencias de los auditores internos gubernamentales chilenos para la obtención de evidencia electrónica de auditoría. En esta investigación, se analizaron aspectos relacionados con la familiaridad y dificultad que tenían los auditores en el manejo de documentos electrónicos, la identificación de soportes y formatos electrónicos y cuestiones relacionadas con la integridad de los documentos electrónicos, entre otras variables.

El trabajo fue realizado sobre 197 auditores internos y los resultados más relevantes fueron que solo el 8,1\% de los auditores gubernamentales cuenta con las competencias para obtener evidencia electrónica de auditoría contable y de calidad.
Teniendo en cuenta los resultados de la investigación, los autores de este estudio plantean la imperiosa necesidad de que los auditores gubernamentales logren urgentemente competencias para obtener evidencia electrónica que permita respaldar sus hallazgos y conclusiones presentadas en los informes de auditoría, para evitar un incremento significativo en el riesgo de auditoría.

La presente investigación va más allá del trabajo realizado por Caldana, Correa \& Ponce (2007), ya que además de analizar el nivel tecnológico de los auditores, en aspectos como la capacidad de detección de evidencia digital, se contrastará ese nivel con el alcanzado por las empresas donde estos profesionales se desempeñan, para determinar si los auditores responden o no, de forma eficiente, a los retos que este ambiente tecnológico requiere. Adicional a ello y en concordancia con los resultados obtenidos, al final del documento se plantean las acciones a 
seguir para optimizar el nivel tecnológico de los auditores, en procura de un mejor desempeño profesional.

No obstante, y teniendo en cuenta el marco teórico y los antecedentes de investigación presentados, no se esperan resultados diametralmente distintos al trabajo de Caldana, Correa \& Ponce (2007), es decir, es poco probable que la competencia digital —o nivel tecnológico- de la muestra analizada de auditores de la ciudad de Bucaramanga resulte positiva, aunque lógicamente, los resultados de los análisis realizados darán luces al respecto.

Los demás trabajos relacionados, aunque abordan temáticas relacionadas con las TIC y la auditoría, sus objetivos y enfoques no están direccionados al objetivo primordial que se aborda en el presente documento: la determinación del nivel tecnológico de los auditores, en oposición al nivel tecnológico de las organizaciones empresariales en las cuales desarrollan sus actividades.

\section{Diseño de la investigación}

La investigación realizada es empírico-descriptiva, ya que su ejecución evidenciará el nivel tecnológico de una muestra de contadores/ auditores de la ciudad de Bucaramanga, lo cual permitirá establecer acciones de mejora para su actualización tecnológica, si así se requiere.

Para lograr la mayor cantidad posible de contadores consultados, se contactó con el Colegio Colombiano de Contadores Públicos, Capítulo Bucaramanga, y con la oficina de Graduados de la Universidad Autónoma de Bucaramanga, de manera que el instrumento (encuesta) fue envia- do a sus bases de datos de correos electrónicos para recolectar la información pertinente.

La colaboración de estos dos organismos permitió que, entre las personas encuestadas, hubiera participación de contadores de la Universidad Autónoma de Bucaramanga - UNAB-, pero también de contadores de otras instituciones universitarias de la ciudad, principalmente, de la Universidad Santo Tomás y de la Universidad Cooperativa de Colombia.

La encuesta aplicada, que buscaba medir el nivel tecnológico de los contadores y de las empresas en las cuales estos se desempeñaban, fue desarrollada teniendo en cuenta los trabajos de Subhasish Dasgupta, Mary Granger y Nina McGarry (2002) y Fabio Gómez (2010), estudios en los cuales se determinó el nivel tecnológico de acuerdo al número de TIC implantadas y utilizadas. En lo que concierne a esta investigación, esta metodología se replicará para determinar las TIC implantadas y utilizadas por los contadores/auditores y por las compañías.

Específicamente, la encuesta está conformada por 36 preguntas, divididas en tres bloques. El primero de ellos hace alusión a los datos de identificación del auditor y de la empresa. El segundo bloque indaga acerca del nivel tecnológico de las compañías y, finalmente, el tercer conjunto de preguntas se refiere al nivel tecnológico de los auditores. Esta encuesta fue cargada en una página web y el enlace de este sitio se envió a las personas consultadas por correo electrónico. Los datos fueron recolectados desde el día 24 de abril y hasta el día 31 de mayo de 2013. Una vez finalizado este período, se logró obtener respuesta de 94 con- 
tadores. Los resultados de estas consultas se presentan en el apartado siguiente.

Algunas de las mediciones que inicialmente se tenía previsto aplicar en el anteproyecto de investigación formulado, tanto para los contadores/auditores como para las empresas, no fueron ejecutadas, por cuestiones de privacidad y seguridad de la información exigida por los contadores y empresas encuestadas.

Independientemente de los resultados obtenidos en la investigación aplicada, es importante aclarar que el objetivo de este trabajo, no se circunscribe simplemente a desnudar unas posibles falencias por parte de los contadores de unas instituciones educativas en particular, sino que busca ser un punto de partida para solucionar una problemática que, en opinión del autor de este escrito, es generalizada en el país, aunque para el contexto de este documento, solo se presentará evidencia de una muestra de profesionales contables en una ciudad específica (Bucaramanga).

\section{Resultados de la investigación}

Teniendo en cuenta el diseño de la encuesta, los resultados obtenidos se presentan en dos bloques: el primero de ellos relacionado con el nivel tecnológico de las empresas y el segundo, relacionado con el nivel tecnológico de los auditores.

Los resultados de la primera de las cuestiones estudiadas evidencian un nivel tecnológico aceptable por parte de las empresas analizadas; la mayoría de empresas consultadas utiliza computadores, tiene una red local, acceso a internet de banda ancha, usa sistemas de correo electrónico, firma electrónica, diversos programas y aplicaciones (contabilidad financiera, gestión comercial y ofimática) y algunos mecanismos de seguridad para la protección de la información, como los antivirus, los cortafuegos y la criptografía, entre otros.

Ahora bien, otros aspectos relacionados con el nivel tecnológico de las empresas presentan comportamientos negativos, es decir, el uso de estas TIC en las compañías analizadas está poco extendido. Ejemplo de lo anterior es el uso de intranet y de sistemas de intercambio electrónico de datos - EDI (Electronic Data Interchange) y la compra y venta de productos por internet.

La variable que obtuvo un mejor comportamiento por parte de las empresas, es la que corresponde al uso de internet, ya que casi el 95\% de las compañías analizadas manifestó usar esta TIC. De otra parte, la variable con peores resultados se refiere a si las empresas ejecutan o no ventas por internet, ya que tan solo el 19\% de las organizaciones empresariales encuestadas realiza esta actividad. Tanto el mejor como el peor resultado son coherentes con otros estudios, que han hecho en Colombia organizaciones como la Comisión de Regulación de Comunicaciones, aunque en el caso de las empresas que venden sus productos o servicios por internet, esta cifra viene mejorando en los últimos años.

En resumen, de las 15 principales variables analizas sobre las compañías, 10 de ellas presentan resultados por encima del 60\% de calificación y 5 de estas variables tienen porcentajes de calificación muy positivos, por encima del 80\%.

Los resultados obtenidos en cuanto al nivel tecnológico de las empresas se presentan en la tabla 1 . 


\begin{tabular}{lccrrrr}
\hline \multirow{2}{*}{ Variable } & \multicolumn{2}{c}{ Sí } & \multicolumn{2}{c}{ No } & \multicolumn{2}{c}{ NS/NR } \\
\cline { 2 - 7 } & Cant. & $\mathbf{( \% )}$ & Cant. & (\%) & Cant. & (\%) \\
\hline Computadores & 85 & 90,4 & 1 & 1,1 & 8 & 8,5 \\
Red local & 73 & 77,7 & 11 & 11,7 & 10 & 10,6 \\
Internet & 89 & 94,7 & 0 & 0,0 & 5 & 5,3 \\
Banda ancha & 82 & 87,2 & 2 & 2,1 & 10 & 10,6 \\
E-mail & 85 & 90,4 & 1 & 1,1 & 8 & 8,5 \\
Firma electrónica & 63 & 67,0 & 21 & 22,3 & 10 & 10,6 \\
Website & 59 & 62,8 & 28 & 29,8 & 7 & 7,4 \\
Servidor seguro & 73 & 77,7 & 9 & 9,6 & 12 & 12,8 \\
Intranet & 40 & 42,6 & 47 & 50,0 & 7 & 7,4 \\
EDI (Electronic Data Interchange) & 30 & 31,9 & 41 & 43,6 & 23 & 24,5 \\
Mecanismos de seguridad & 83 & 88,3 & 5 & 5,3 & 6 & 6,4 \\
Programas y aplicaciones & 68 & 72,3 & 16 & 17,0 & 10 & 10,6 \\
Compra por internet & 36 & 38,3 & 42 & 44,7 & 16 & 17,0 \\
Vende por internet & 18 & 19,1 & 65 & 69,1 & 11 & 11,7 \\
Controles computarizados & 44 & 46,8 & 40 & 42,6 & 10 & 10,6 \\
\hline
\end{tabular}

Tabla 1. Resultados variables nivel tecnológico de las empresas Fuente: elaboración propia

El segundo bloque de análisis se refería al nivel tecnológico de los auditores. Al respecto, los resultados obtenidos son claramente negativos. En ninguna de las 12 variables estudiadas se evidenciaron buenos resultados; de hecho, es todo lo contrario. Según la información resultante, los auditores encuestados, en su mayoría, no conocen las técnicas automatizadas de auditoría, ni las técnicas de fraude informático, poco utilizan herramientas para la protección de la información o software de auditoría, menos de la mitad de los auditores utiliza la estadística en el desarrollo de sus labores y un bajo porcentaje de los profesionales encuestados $(23,4 \%)$ ha realizado auditoría de sistemas, alguna vez en su vida profesional. Este tipo de auditoría no puede estar por fue- ra del interés del contador público, teniendo en cuenta el entorno tecnológico actual, en el cual se desarrolla la mayoría de las organizaciones empresariales.

La variable que obtuvo un mejor comportamiento por parte de los profesionales contables, fue la utilización de herramientas informáticas para la protección de la información, pero infortunadamente, con apenas un 45,7\% de calificación. De otra parte, la variable con peores resultados se refiere a si los contadores/ auditores se sienten capacitados para el reconocimiento de evidencia digital, variable que obtuvo una medición del 19,1\%.

El resumen de los resultados obtenidos en cuanto al nivel tecnológico de los contadores/ auditores se presenta en la tabla 2 . 


\begin{tabular}{|c|c|c|c|c|c|c|}
\hline \multirow{2}{*}{ Variable } & \multicolumn{2}{|c|}{ Sí } & \multicolumn{2}{|c|}{ No } & \multicolumn{2}{|c|}{ NS/NR } \\
\hline & CANT. & (\%) & CANT. & $(\%)$ & CANT. & (\%) \\
\hline Conoce técnicas automatizadas de auditoría & 28 & 29,8 & 64 & 68,1 & 2 & 2,1 \\
\hline Utiliza técnicas automatizadas de auditoría & 25 & 26,6 & 59 & 62,8 & 10 & 10,6 \\
\hline Utiliza herramientas para proteger la información & 43 & 45,7 & 44 & 46,8 & 7 & 7,4 \\
\hline Conoce técnicas de fraude informático & 37 & 39,4 & 53 & 56,4 & 4 & 4,3 \\
\hline Utiliza software para la auditoría & 19 & 20,2 & 66 & 70,2 & 9 & 9,6 \\
\hline Aplica pruebas de auditoría & 24 & 25,5 & 60 & 63,8 & 10 & 10,6 \\
\hline Ha realizado auditoría de sistemas & 22 & 23,4 & 64 & 68,1 & 8 & 8,5 \\
\hline Está capacitado en reconocimiento de evidencia digital & 18 & 19,1 & 66 & 70,2 & 10 & 10,6 \\
\hline Usa estadística en trabajo de auditoría & 41 & 43,6 & 41 & 43,6 & 12 & 12,8 \\
\hline Usa muestreo aleatorio en trabajo de auditoría & 42 & 44,7 & 38 & 40,4 & 14 & 14,9 \\
\hline Conoce métodos de muestreo aleatorio & 22 & 23,4 & 53 & 56,4 & 19 & 20,2 \\
\hline Usa métodos de muestreo aleatorio & 19 & 20,2 & 49 & 52,1 & 26 & 27,7 \\
\hline
\end{tabular}

Tabla 2. Resultados variables nivel tecnológico de los contadores/auditores Fuente: elaboración propia

A partir de los resultados presentados anteriormente, se generan varias conclusiones y recomendaciones sobre el nivel tecnológico de las empresas y del nivel tecnológico de los contadores/auditores analizados. Estas conclusiones y recomendaciones serán descritas en el siguiente apartado.

\section{Conclusiones y recomendaciones}

Una de las primeras conclusiones que se puede extraer de forma inmediata, teniendo en cuenta los resultados obtenidos en la investigación, es que el nivel tecnológico de los contadores y auditores no está en concordancia con el nivel tecnológico de las empresas, es decir, los contadores y auditores analizados no están capacitados ni tienen la capacidad tecnológica suficiente para responder a los retos que demanda el ambiente tecnológico en el cual se desempeñan las empresas objeto de estudio, compañías que demuestran un razonable y positivo nivel tecnológico, pero que al mismo tiempo están expuestas a una serie de riesgos, que son consecuencia del uso de TIC, los cuales no están siendo ni siquiera identificados por los contadores $\mathrm{y}$ auditores.

En términos generales, el nivel tecnológico de las empresas analizadas es bueno, como ha quedado evidenciado en el apartado de resultados del presente documento. Estos resultados parecen demostrar que las compañías analizadas de la ciudad de Bucaramanga están conscientes de la importancia de invertir recursos en la adopción y uso de tecnologías de la información y la comunicación en el desarrollo de sus actividades diarias, inversiones que según lo expuesto por Fabio Gómez (2010) y Pedro Lorca-Fernández y Juan de Andrés-Suárez (2007), 
rendirán sus frutos en el mediano y largo plazo, aunque también es importante anotar que el uso o no de TIC en las organizaciones empresariales dependerá también de las características y necesidades de cada compañía.

No se puede llegar a las mismas conclusiones de acuerdo a los resultados obtenidos para el caso del nivel tecnológico de los contadores/ auditores. Los resultados confirman, al menos en la muestra analizada, el imaginario histórico que se ha tenido acerca de los contadores públicos en este sentido, suposición que indica que a los contadores públicos no les interesa la aplicación de TIC en su desempeño profesional, al menos en lo que se refiere a la ejecución de trabajos de auditoría, con los efectos funestos que tal situación tiene para la calidad del trabajo desarrollado por estos profesionales y, en consecuencia, los resultados negativos que supone para las empresas auditadas esta falta de conocimientos tecnológicos.

Mención especial requieren los resultados obtenidos en cuanto a la capacidad que tienen los contadores/auditores de reconocer evidencia digital de auditoría, pues son muy deficientes, situación que resulta ampliamente preocupante cuando hoy la gran mayoría de actividades empresariales se lleva a cabo con tecnologías de la información y la comunicación y muchos de los fraudes que se cometen en las empresas se ejecutan precisamente con las TIC y los sistemas de información automatizados.

De hecho, se podría plantear que las tecnologías de la información y la comunicación existentes y que los contadores y auditores tienen a su disposición, dejan de ser un soporte válido para la ejecución de su trabajo y se con- vierten en una carga que les dificulta la realización de sus actividades, no porque las TIC supongan un problema en sí, sino simplemente porque los profesionales contables no están preparados para utilizarlas.

No tiene sentido perder tiempo en buscar las razones del desinterés de los contadores y auditores de la muestra analizada en las TIC. Independientemente de la causa de tal situación, lo importante es subsanar estas debilidades con programas de actualización y formación que "ataquen y eliminen" estas falencias identificadas mediante la investigación efectuada.

Esta actualización y esta formación pueden llevarse a cabo mediante la inserción de asignaturas o módulos en los programas de pregrado y posgrado que aborden estas temáticas $\mathrm{o}$, incluso, mediante el diseño y formulación de diplomados o cursos de corta duración, por fuera de los planes de estudios de los programas de pregrado y posgrados de las facultades de Contaduría Pública; tales cursos se enfocarían específicamente en contenidos relacionados con las tecnologías de la información y la comunicación aplicables en los procesos y trabajos de auditoría desarrollados por los contadores, para dar respuesta a las necesidades y debilidades encontradas en esta investigación.

Lógicamente, este esfuerzo deberá hacerse con el concurso de los programas o facultades de Contaduría Pública en el país y, en la medida de lo posible, con la participación de organismos contables de carácter nacional, como el Consejo Técnico de la Contaduría Pública, para que haya una entidad rectora de la profesión contable en el país, que oriente el desarrollo de estos 
procesos de formación y educación, como se está realizando con las Normas Internacionales de Información Financiera y las Normas Internacionales de Aseguramiento de la Información.

Finalmente, en la realización de esta investigación hubo dos importantes dificultades que limitaron los resultados obtenidos en el trabajo. En primera instancia, la apatía en la participación de los contadores, auditores y empresas en el trabajo de campo, lo cual redujo la cantidad de información recolectada. Y, en segundo término, es fundamental indicar que los profesionales contables y empresas que participaron en el estudio, exigieron una serie de garantías en cuanto a la seguridad y confidencialidad de la información, con lo cual los análisis y resultados que se lograron obtener con la información resultante, se vieron afectados y limitados.

A pesar de que estos resultados no pueden ser extrapolados a la totalidad de población de contadores y auditores de la ciudad de Bucaramanga y, mucho menos del país, es un punto de partida o de diagnóstico, con el cual la comunidad académica y gremial puede empezar a trabajar en procura de mejorar la calidad de los servicios ofrecidos y prestados por los contadores públicos, en beneficio de las organizaciones empresariales.

Igualmente, para futuras investigaciones relacionadas con la temática desarrollada en este trabajo, sería posible ampliar la población analizada a diferentes ciudades del país y la cantidad de variables estudiadas, y se podría profundizar en las características y atributos personales y profesionales de los contadores/auditores, para encontrar patrones de comportamiento, de tal forma que se pudiera establecer una idea más clara y concisa acerca de su nivel tecnológico. Esos resultados confirmarían o refutarían las conclusiones planteadas en este documento y permitirían abordar el diseño de soluciones al problema objeto de estudio, con un mayor grado de seguridad.

En opinión del autor de este escrito, es de esperar que investigaciones más amplias y profundas en el país lleguen probablemente a los mismos resultados y conclusiones, lo cual no deja de ser preocupante para el futuro de la profesión contable en Colombia.

\section{Referencias}

Barua, Anitesh; Kriebel, Charles \& Mukhopadhyay, Tridas (1995). Information Technology and Business Value: An Analytic and Empirical Investigation. Information Systems Research, 6 (1), 3-23.

Benvenuto-Vera, Ángelo (2004). Los delitos informáticos (Ley 19.223 Chile) y la función de auditoría informática. CAPIC Review, Conferencia Académica Permanente de Investigación Contable, 2, 27-34. Disponible en: file:///D:/Mis\%20documentos/Downloads/Dialnet-LosDelitosInformaticosLey19223ChileYLaFuncionDeAud-2573421.pdf Bonsón, Enrique (2000). La auditoría de cuentas en la economía digital: hacia un nuevo paradigma. Revista de la Asociación Española de Contabilidad y Administración de Empresas, AECA, 52, 56-59.

Brynjolfsson, Erik (1993). The Productivity Paradox of Information Technology. Communications of the ACM, 36 (12), 67-77. 
Disponible en: http://ccs.mit.edu/papers/ CCSWP130/CCSWP130.html

Caldana Daniella; Correa, Ricardo \& Ponce, Héctor (2007). Competencias de los auditores gubernamentales chilenos para la obtención de evidencia electrónica de auditoría. Revista Contaduría y Administración, Universidad de Santiago de Chile, 223, 9-31. Disponible en: http://www.scielo.org.mx/pdf/cya/ n223/n223a2.pdf

Católico, Diego \& Gómez, Fabio (2013). Transparencia informativa on-line en empresas colombianas. Revista Internacional Legis de Contabilidad y Auditoría, 55, julio-septiembre, 93-118.

Colombia (2000). Ley 599 de 2000, por la cual se expide el Código Penal. Diario Oficial, 44.097, 24 de julio de 2000. Disponible en: http://www.secretariasenado.gov.co/senado/ basedoc/ley/2000/ley_0599_2000.html.

Colombia (2009). Ley 1314 de 2009, por la cual se regulan los principios y normas de contabilidad e información financiera y de aseguramiento de información aceptados en Colombia, se señalan las autoridades competentes, el procedimiento para su expedición y se determinan las entidades responsables de vigilar su cumplimiento. Diario Oficial, 47.409, 13 de julio de 2009. Disponible en: http:/www.secretariasenado.gov.co/senado/ basedoc/ley/2009/ley_1314_2009.html

Dasgupta, Subhasish; Granger, Mary \& McGarry, Nina (2002). User Acceptance of E-Collaboration Technology: An Extension of the Technology Acceptance Model. Group Decision and Negotiation, 11 (2), 87-100. Disponible en: http://home.gwu. edu/ dasgupta/pubs/2002-gdn-dasguptaet-al.pdf

García-Benau, María Antonia \& Vico-Martínez, Antonio (2003). Los escándalos financieros y la auditoría: pérdida y recuperación de la confianza en una profesión en crisis. Revista Valenciana de Economía y Hacienda, 7, 25-48.

Gómez, Fabio (2010). La factura electrónica en las empresas aragonesas: perfil financiero tecnológico y efectos de la implantación. Tesis doctoral, Departamento de Contabilidad y Finanzas, Universidad de Zaragoza, Aragón, España. Disponible en: http://zaguan.unizar. es/record/4819/files/TESIS-2010-044.pdf

Gómez, Fabio \& Católico, Diego (2009). Revelación y divulgación de la información financiera y no financiera on-line de las 500 empresas más representativas en Colombia. Cuadernos de Contabilidad, 10 (27), 269318. Disponible en: http://www.javeriana. edu.co/fcea/cuadernos_contab/vol10_n_27/ vol10_27_3.pdf

Gubba, Hugo; Gutfraind, Jorge; Rodríguez, Rubén \& Villamarzo, Ricardo (2001). Auditoría de evidencia virtual: cambio filosófico o adaptación del profesional a las nuevas modalidades. Revista de Antiguos Alumnos del Instituto de Estudios Empresariales de Montevideo, IEEM, 4 (1), 32-44.

International Federation of Accountants, IFAC (2009a). International Education Standards - IES 2: Content of Professional Accounting Education Programs. Disponible en: https://www.ifac.org/sites/default/ files/publications/files/ies-2-content-ofprofessi.pdf 
International Federation of Accountants, IFAC (2009b). International Standard on Auditing 315: Identifying and Assessing the Risk of Material Misstatement through Understanding the Entity and its Environment. Disponible en: http://www.ifac.org/sites/default/files/ downloads/a017-2010-iaasb-handbookisa-315.pdf

Llopart, Xavier (1997). Gestión de los recursos humanos en base a competencias. Análisis de competencias en empresas de auditoría. Tesis doctoral, Departamento de Economía y Organización de Empresas, Universitat de Barcelona, España. Disponible en: http://www.tesisenred.net/handle/10803/1481

Lorca, Pedro \& De Andrés, Javier (2007). Efectos de la implantación de sistemas integrados de gestión (ERP) en las grandes empresas españolas. Revista Española de Financiación y Contabilidad, 36 (135), 595-

623. Disponible en: file:///D:/Mis\%20 documentos/Downloads/Lorca_Suarez_2007_REFC.pdf

Luo, Wenhong; Cook, David; Joseph, Jimmie \& Ganapathy, Bopana (2000). An Exploratory Framework for Understanding Electronic Bill Presentment and Payment -EBPP_ Model Selection. Human Systems Management, 19 (4), 255-264.

Martínez, María del Carmen \& Armenteros, Ileana (2006). Orígenes y clasificación de la auditoría de la información. Revista Acimed, 14 (5). Disponible en: http://
scielo.sld.cu/scielo.php?pid=S102494352006000500017\&script=sci_arttext Ojeda, Jorge; Rincón, Fernando; Arias, Miguel \& Daza, Libardo (2010). Delitos informáticos y entorno jurídico vigente en Colombia. Cuadernos de Contabilidad, 11 (28), 41-66. Disponible en: http://revistas. javeriana.edu.co/index.php/cuacont/article/ view/3176/2416

Pinsonneault, Alain \& Rivard, Suzanne (1998). Information Technology and the Nature of Managerial Work: from Productivity Paradox to the Icarus Paradox. MIS Quarterly, 22 (3), 287-311.

Porter, Thomas \& Burton, John (1980). Auditoría: un análisis conceptual. México: Diana.

Roach, Stephen S. (1991). Services under Siege: the Restructuring Imperative. Harvard Business Review, 68, 82-91.

Valderrama, Marcos (2002). ERP y lo contable. Working Paper. Pontificia Universidad Javeriana. Departamento de Ciencias Contables, Bogotá.

- Fecha de recepción: 26 de agosto de 2013

- Fecha de aceptación: 14 de marzo de 2014

\section{Para citar este artículo}

Gómez-Meneses, Fabio Enrique (2014). Competencia digital en la auditoría. Soporte o carga en el ejercicio profesional de los auditores. Cuadernos de Contabilidad, 15 (37), 135-151. 
